\title{
Heartbeat: Prevention of sudden cardiac death
}

Primary prevention of sudden cardiac death (SCD) with an implantable cardioverter-defibrillator (ICD) now is a key element of clinical care in adults with heart failure with reduced ejection fraction (HFrEF). However, risk stratification is needed to ensure that a specific patient with HFpEF will benefit from an ICD because these devices are expensive and may cause harm, including inappropriate shocks. The National Institute for Health and Care Excellence (NICE) introduced new guidelines (TA314) for implantable cardioverter-defibrillators (ICD) in 2014.

In this issue of Heart, Cubbon and colleagues (see page 735) evaluated the prognostic accuracy of the TA314 NICE guidelines in a prospective multicenter cohort of 1026 patients with chronic stable HFrEF (EF $\leq 45 \%$ ). The overall risk of SCD or an appropriate ICD shock was 2.1 events per 100 patient-years (95\% CI 1.7 to 2.6) with $31.1 \%$ of patients meeting TA314 ICD criteria. Compared to those who did not meet criteria, SCD or an appropriate ICD shock was 2.5 times (95\% CI 1.6 to 3.9) more likely in patients meeting TA314 ICD criteria. In addition, these patients had an increased risk of death due to progressive heart failure (HR 1.6, 95\% CI 1.1 to 2.3 ) or to non-cardiovascular causes (HR 1.5, 95\% CI 1.1 to 2.2). However, risk for diabetic patients who did not meet TA314 ICD criteria was similar to non-diabetics who did meet criteria. Similarly, risk for patients with an ischemic cardiomyopathy who did not meet criteria was similar to those with non-ischemic cardiomyopathy who did meet TA314 ICD criteria (figure 1). These findings were robust on sensitivity analyses. Based on these findings, the authors suggest that "Future updates to TA314 should consider refining risk-stratification approaches to minimise both preventable sudden death and overtreatment".

In the accompanying editorial, Professor Cowie (see page 731) summarizes the treatment options (table 1) in the TA314 guidelines relevant to patients with HFpEF. He points out that: "within [Cubbon and colleagues] study

Correspondence to Professor Catherine M Otto, Division of Cardiology, University of Washington, Seattle, WA 98195, USA; cmotto@u.washington.edu population, the absolute risk of SCD was heavily influenced by the presence of diabetes mellitus and ischaemic heart disease - so the event rates in patients with diabetes (or ischaemic heart disease) not meeting the specific TA314 criteria (due to a narrow QRS complex) were similar to patients without diabetes (or ischaemic heart disease) who did. This highlights a flaw in the NICE guidance-although the recommended approach works well to identify patients at high relative risk of SCD, it does not provide a precise estimate of an individual's absolute risk, and presumably, therefore, the likely absolute benefit. If the absolute benefit is not considered, then the physician and patient may have an unrealistic estimate of the potential benefit of ICD technology, and NHS resources may be used inappropriately".
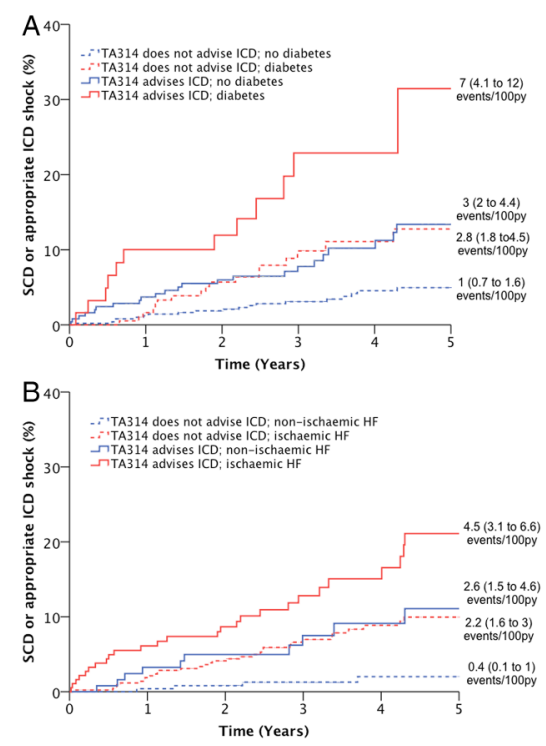

Figure 1 Performance of standard TA314 criteria in patients with diabetes or ischaemic aetiology. Kaplan-Meier curves demonstrating that within populations stratified by (A) diabetes or (B) ischaemic aetiology, TA314 identifies subgroups at increased relative risk of sudden death or appropriate ICD shock $(p<0.05$ for all within stratum comparisons of TA314). However, absolute event rates in subgroups of patients meeting TA314 criteria were similar to those in subgroups not meeting TA314 criteria, depending on the presence of diabetes or ischaemic aetiology. $H F$, heart failure; ICD, implantable cardioverter-defibrillator; SCD, sudden cardiac death.
Another approach to prevention of sudden cardiac death, particularly in people without a previous history of heart disease, is widespread training in cardiopulmonary resuscitation and public access to automated external defibrillators (AEDs). An important barrier to use of AEDs is being able to quickly locate a nearby AED when needed. To ensure that people can find the AED, the International Liaison Committee on Resuscitation (ILCOR) developed a universal sign for AEDs based on internal standards for safety signs in 2008. However, in a survey of 493 travelers from 42 countries at Copenhagen airport, Aagaard and colleagues (see page 770) found that only 39\% correctly identified the ILCOR AED sign (figure 2). They conclude that "Initiatives promoting public awareness of AEDs are warranted."

My conclusion is somewhat different. No wonder so few recognized the "universal" AED sign - before reading this article I was not aware there was a universal sign so maybe others are unaware as well. Further, I see many different AED signs at home and when traveling around the world, but have rarely seen this one. How can we possibly expect a member of the general public to recognize these signs or even to know what "AED" means? A quick Google image search shows a lot of

Table 1 Treatment options with ICD or CRT for people with heart failure who have left ventricular dysfunction with an LVEF of $35 \%$ or less (according to NYHA Class, QRS duration and the presence of LBBB)

\begin{tabular}{|c|c|c|c|c|}
\hline \multirow[b]{2}{*}{ QRS interval } & \multicolumn{4}{|c|}{ NYHA Class } \\
\hline & I & II & III & IV \\
\hline$<120 \mathrm{~ms}$ & $\begin{array}{l}\text { ICD if } \\
\text { risk of } \\
\text { death }\end{array}$ & $\begin{array}{l}\text { there is a } \\
\text { sudden }\end{array}$ & $\begin{array}{l}\text { high } \\
\text { cardiac }\end{array}$ & $\begin{array}{l}\text { ICD and } \\
\text { CRT not } \\
\text { clinically } \\
\text { indicated }\end{array}$ \\
\hline $\begin{array}{l}120-149 \mathrm{~ms} \\
\text { without LBBB }\end{array}$ & ICD & $I C D$ & ICD & CRT-P \\
\hline $\begin{array}{l}120-149 \mathrm{~ms} \\
\text { with LBBB }\end{array}$ & $I C D$ & CRT-D & $\begin{array}{l}\text { CRT-P } \\
\text { or } \\
\text { CRT-D }\end{array}$ & CRT-P \\
\hline $\begin{array}{l}\geq 150 \text { ms with } \\
\text { or without } \\
\text { LBBB }\end{array}$ & CRT-D & CRT-D & $\begin{array}{l}\text { CRT-P } \\
\text { or } \\
\text { CRT-D }\end{array}$ & CRT-P \\
\hline
\end{tabular}

Adapted from TA314. CRT, cardiac resynchronisation technology; LBBB, left bundle branch block; LVEF, left ventricular ejection fraction; NYHA, New York Heart Association. 


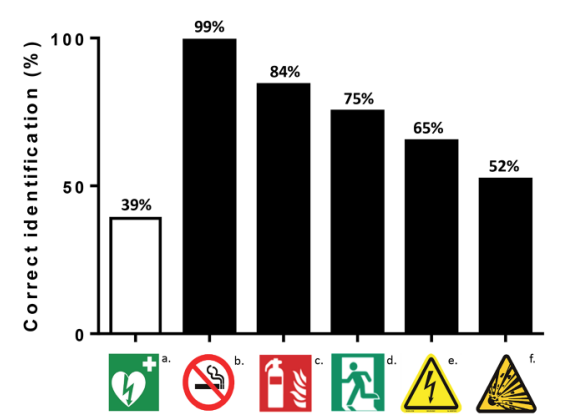

Figure 2 Correct interpretation of safety signs. Numbers above bars indicate correct interpretation as percentage of total. (a) International Liaison Committee on Resuscitation automated external defibrillator sign, (b) no smoking, (c) fire extinguisher, (d) emergency exit, (e) high voltage and ( $f$ ) danger of explosion.

variation in AED sign color and design, although most have some variation of a valentine and lightning bolt. Surely, signs work best when the graphics are so intuitive that everyone, regardless of language or culture, immediately understands what they mean. Maybe we need a better sign for public access defibrillators, possibly a better name too.

The optimal duration of dual antiplatelet therapy (DAPT) in patients with acute coronary syndromes and the role of DAPT in other coronary disease presentations continues to be an area of active inquiry. A small randomized double-blind placebo controlled clinical trial by Saw and colleagues (see page 763) showed that the addition of ticagrelor to aspirin after coronary artery bypass graft surgery reduced graft occlusion as assessed by computed tomographic angiography 3 months after surgery. Ticagrelor therapy was a univariate and multivariate predictor of graft occlusion suggesting that larger clinical trials of this therapy should be considered.

A review article by Parker and Storey (see page 783) addresses long-term dual antiplatelet therapy (DAPT) following myocardial infarction focusing on the implications of the PEGASUS-TIMI 54 Study (Prevention of Cardiovascular Events in Patients with Prior Heart Attack Using Ticagrelor Compared to Placebo on a Background of Aspirin - Thrombolysis in Myocardial Infarction 54) (figure 3). They conclude that "Overall, the PEGASUS-TIMI 54 results demonstrate that patients with a history of acute coronary syndrome deemed to be at high risk of further ischaemic events, particularly those in whom the risks of ischaemic events and cardiovascular death outweigh the risk of life-threatening bleeding, may benefit from prolonged ticagrelor-based DAPT."

The Education in Heart article in this issue (see page 796) provides a nice summary of new diagnostic approaches in infective endocarditis. Microbiological cultures identify about $80 \%$ of cases; in the remainder molecular methods may be considered, using DNA extraction with PCR amplification and sequence confirmation to identify the causative organism. Positron emission tomography (PET) combined with computed tomographic (CT) imaging may help detect areas of infection or inflammation in patients with prosthetic valves or implanted cardiac electronic devices. These new approaches are additive to the standard Duke criteria for diagnosis of endocarditis.

See if you can make the diagnosis from the ECG of an an 88-year-old man with syncope and an alternating QRS axis (see page 769 ). There now are over 60 online Image Challenge cases with multiple choice questions and detailed explanations of the correct answer. Look under the Topic Collections in the Archives tab on the Heart homepage (http://heart.bmj. $\operatorname{com} /$ ) and then select "Image Challenges". These Image Challenges are a great way to test your diagnostic skills or to improve your imaging knowledge base.

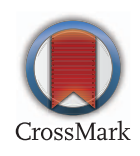

To cite Otto CM. Heart 2016;102:729-730.

Heart 2016;102:729-730.

doi:10.1136/heartjnl-2016-309722

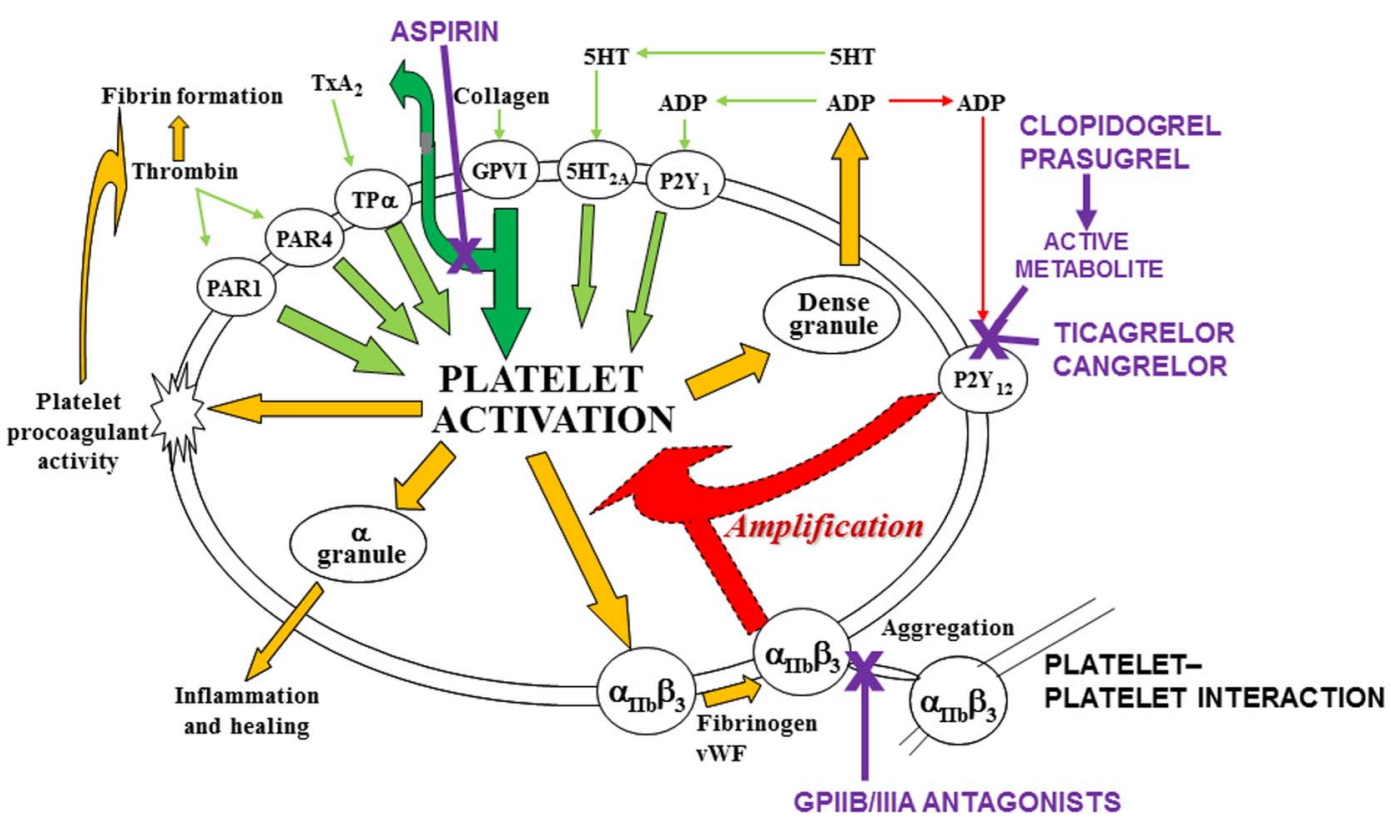

Figure 3 Mechanisms of platelet activation and targets of antiplatelet drugs. Aspirin blocks the formation of thromboxane A2 (TxA2), which plays an important but limited role in platelet activation. P2Y12 inhibitors block a central amplification pathway that has profound effects on platelet responses, amplifying the responses to all the key platelet activation pathways. Modified with permission from Storey RF. Biology and pharmacology of the platelet P2Y12 receptor. Curr Pharm Des 2006;12:1255-9. 